\title{
The Stockholm spinal cord injury study: 1. Medical problems in a regional SCI population
}

\author{
R Levi ${ }^{1,4}, \mathrm{C}$ Hultling ${ }^{1}, \mathrm{MS}$ Nash $^{1-3}$ and $\AA$ Seiger ${ }^{1,3,4}$ \\ ${ }^{1}$ Solberga Spinal Cord Injury Research Project, Karolinska Institute; ${ }^{2}$ Division of Physical Therapy, Department \\ of Orthopaedics \& Rehabilitation; ${ }^{3}$ The Miami Project to Cure Paralysis, University of Miami School of \\ Medicine, Miami, Florida, USA; ${ }^{4}$ Department of Clinical Neuroscience and Family Medicine, Karolinska \\ Institute, Huddinge University Hospital, Stockholm, Sweden
}

\begin{abstract}
Out of a regional traumatic spinal cord injury population consisting of 379 individuals, 353 (93.1\%) participated in the present study. Subjects were individually interviewed using semi-structured protocols. In addition, previous medical records were available for over $96 \%$ of subjects, and were used in all these cases to minimise recall bias. Cause of injury, prevalence of present medical symptoms and occurrence of medical complications in the post-acute, post-discharge phase were recorded. Neurological classification was verified by physical examination according to ASIA/IMSOP standards. Many subjects had experienced complications since discharge from initial hospitalisation, especially urinary tract infections, decubitus ulcers, urolithiasis, and neurological deterioration. Prevalence of medical symptoms was also high. More than $41 \%$ of subjects with spastic paralysis reported excessive spasticity to be associated with additional functional impairment and/or pain. Almost two-thirds of subjects reported significant pain, with a predominance of neurogenic-type pain. Bladder and bowel dysfunction were each rated by nearly $41 \%$ of subjects as a moderate to severe life problem. As expected, sexual dysfunction was also commonly reported. Prevalence of reported symptoms by general systems review was high, particularly fatigue, constipation, ankle oedema, joint and muscle problems, and disturbed sleep. However, lack of adequate normative data precludes comparison with the general population. The frequent occurrence of reported medical problems and complications support advocacy of comprehensive, life-long care for SCI patients. The commonly reported problems of neurogenic pain and neurological deterioration, in particular, require more attention, as these symptoms are not seldom ominous, either by virtue of their impact on quality of life, or because of underlying pathology.
\end{abstract}

Keywords: spinal cord injury; epidemiology; outcome; complications; neurological deterioration

\section{Introduction}

The body of knowledge regarding long-term consequences of traumatic spinal cord injury (SCI) has expanded considerably in the post World War II era, most notably because of the pioneering work of Sir Ludwig Guttmann and co-workers at the National Spinal Injuries Centre, Stoke Mandeville Hospital, ${ }^{1}$ as well as through information derived from the SCI database initiated in 1972 under the jurisdiction of the United States Rehabilitation Services Administration (now the National Institute for Disability and Rehabilitation Research). ${ }^{2,3}$ Aging of the SCI population has specifically been addressed in a publication, ${ }^{4}$ which serves to forewarn that spinal man is susceptible to

Correspondence: R Levi, Solberga SCI Research Project, Karolinska Institute S-126 91, Stockholm, Sweden many unique, life-long medical and social challenges. If these challenges remain unknown, are misinterpreted, or understood but not acted upon, additional impairment, disability, and handicap may result. Therefore, reliable reporting of the medical problems concerning SCI populations is essential.

Several papers have addressed the characteristics of injury and rehabilitative outcomes for survivors of $\mathrm{SCI}^{5-7}$ Studies addressing medical problems among chronic SCI subjects, however, are often limited by selection bias, such as high dropout-rates or a priori highly selected study populations. The Swedish tradition of socialised medicine, as well as a high degree of registration of its citizens, facilitates subject identification, and has provided the opportunity for a survey of the regional Stockholm SCI population, the largest in Sweden. The analysis of medical problems, a subset of this survey, is presented. 


\section{Methods}

Permission to establish a computerised health care data-base was granted by the regional authorities according to Swedish jurisdiction.

\section{Subject identification}

The study group comprised persons with SCI of traumatic etiology, living within the primary catchment area for the Stockholm Regional SCI Centre (under planning at the time of the study). The region has a total population of approximately 1.67 million. ${ }^{8}$ Subjects were identified in three stages.

\section{Stage 1 Survey of regional registers:}

- files from all intraregional hospitals with SCI outpatient services

- files from all regional SCI patient organisations and private regional SCI rehabilitation facilities

- files from the regional data bank, containing registration of all-cause inpatient treatment in all intraregional hospitals since 1975 (onset of comprehensive registration).

Stage 2 All subjects identified in stage 1 were contacted by the research staff, whereby the diagnosis of traumatic SCI was confirmed by the subject.

Stage 3 All subjects with confirmation of definite or likely traumatic SCI by the stage 2 procedure were invited to the Solberga SCI research project for participation in the study. Definite confirmation of correct diagnosis was made at this time by three methods: (1) review of medical records, (2) interview, and (3) physical examination of subjects.

\section{Definitions}

In describing the SCI study group, the following terms are used in all tables and connecting text:

complication: a medical condition associated with the SCI, diagnosed by a physician

symptom: self-reported dysfunction and/or discomfort problem: complications and/or symptoms.

\section{Data collection}

The study was conducted by individual sessions, where each subject met with a physician, a nurse and a social worker on the same day. Typically, 3-4 h were spent with each subject taking medical and social history and performing the physical examination. The staff also administered assessment forms regarding quality of life and living conditions, and collected blood and urine samples. However, only data pertinent to the subject of this paper, ie the causes of injury, neurological status, and post-acute medical problems, are reported herein. Data were obtained by direct questioning of the subjects, combined with review of previous medical records, the latter source being used to corroborate the information given by the subject. Medical records were available for $96.3 \%$ of all subjects. On rare occasions, the patient's history and the medical records provided inconsistent or contradicting data. Typically, these situations were solved by direct contact with the treating clinic, by obtaining additional medical records, or both.

Standardisation of interviews was imposed by semistructured protocols defining the minimum information required for each subject, also allowing for more detailed descriptions when available. Medical terminology used in these protocols was clarified to the patient. Subjective statements, such as whether a certain factor (eg pain) constituted a 'significant' problem for the patient, were given a standardised operational definition.

A detailed description of this now computerised database has been previously published..$^{9}$

Neurological examination was performed and documented according to American Spinal Injury Association/International Medical Society of Paraplegia (ASIA/IMSOP) standards, 1992 revision. $^{10}$

Data collection was performed at the Solberga SCI Research Project from November 1991 to June 1994, with most subjects being examined during 1992-1993.

\section{Results}

Three hundred and seventy four subjects out of the 400 verified by the stage 2 search participated in the study. Twenty six persons either refused to participate, or did not show up for interview despite several bookings. Medical records for these 26 subjects were obtained, and the diagnosis of traumatic SCI was judged appropriate in all the cases. Among the 374 that were examined, 21 were found to have diagnoses other than traumatic SCI and were excluded from further analysis. Thus, the number of identified subjects with traumatic SCI within the catchment area was 379 . The study group comprised 353 subjects, making the dropout rate $6.9 \%$.

All data are expressed as numerical outcomes and percentages. Descriptive characteristics of the subjects regarding age at injury, are depicted in Table 1 . Two hundred and eighty six subjects $(81 \%)$ were male, and 67 were female. Mean age at injury, as well as mean age at contact, were almost similar for both sexes.

International Classification of Diseases (ICD) and classification of external causes of injury, ${ }^{11}$ for our patient group, are reported in Table 2. Gross neurological level of lesion (NLL) and completeness of injury are shown in Table 3.

\section{Medical complications}

Table 4 shows the number of subjects who had experienced a given complication at least once after discharge from initial hospitalisation. All medical complications specified in the table were explicitly sought. Definitions were given as specified in the legend. In addition, subjects were asked for occurrence of 'any other complications'. The most frequently occurring conditions in the latter group were: bacterial 
Table 1 Demographic data

\begin{tabular}{|c|c|c|}
\hline \multirow{2}{*}{$\begin{array}{l}\text { Age at contact } \\
\text { (years) }\end{array}$} & \multicolumn{2}{|c|}{ Total sample $n=353$} \\
\hline & No. & $\%$ \\
\hline $\begin{array}{r}0-15 \\
16-30 \\
31-45 \\
46-60 \\
61+\end{array}$ & $\begin{array}{r}2 \\
79 \\
133 \\
95 \\
44\end{array}$ & $\begin{array}{r}0.6 \\
22.4 \\
37.7 \\
26.9 \\
12.5\end{array}$ \\
\hline $\begin{array}{l}\text { Mean (years) } \\
\text { Standard deviation } \\
\text { Range } \\
\text { Median }\end{array}$ & \multicolumn{2}{|c|}{$\begin{array}{c}42 \\
14 \\
11-78 \\
41\end{array}$} \\
\hline Age at injury (years) & No. & $\%$ \\
\hline $\begin{array}{r}0-15 \\
16-30 \\
31-45 \\
46-60 \\
61+\end{array}$ & $\begin{array}{r}18 \\
184 \\
93 \\
51 \\
7\end{array}$ & $\begin{array}{r}5.1 \\
52.1 \\
26.3 \\
14.4 \\
2.0\end{array}$ \\
\hline $\begin{array}{l}\text { Mean } \\
\text { Standard deviation } \\
\text { Range } \\
\text { Median }\end{array}$ & & \\
\hline
\end{tabular}

cellulitis (10), osteomyelitis (9), surgery for bowel obstruction (7), severe skin burns (6), epididymitis (6), and major depressive episodes (5).

\section{Prevalence of all-cause progressing and/or new neurological symptoms}

Progressing and/or new neurological symptoms in Table 5 refer to the worsening, or de novo occurrence, of one or more of the following symptoms: motor loss (at, above or below the NLL), sensory loss (at, above or below the NLL), pain (any type, severity and location), spasticity, autonomic dysreflexia (either verified by physician or suspected because of typical symptoms) and hyperhidrosis (any location). Subjects were instructed to disregard symptoms that had been present since the initial hospitalisation, unless they had experienced any worsening.

\section{Prevalence of pain}

Subjects were told to disregard pain of short duration, and only indicate longer-standing, 'chronic', pain. However, no specific time limit was given. Subjects having such pain were then asked if they perceived this to be a 'significant problem', being operationally defined as 'having a continuous negative impact on your quality of life'.

Table 4 depicts the occurrence of significantly problematic pain in the study population. Those confirming pain as a problem by this definition were then asked whether pain was their 'primary problem', when considering all aspects of their spinal cord injury.

A detailed pain history was elicited. The physician then classified the pain as either neurogenic or nonneurogenic. Pain of a burning, stabbing, or sharp shooting quality segmentally at, or diffusely below, the NLL was considered neurogenic, whereas for example load- or position-related pain of a throbbing or aching quality above the NLL was classified as non-neurogenic (Table 6).

\section{Prevalence of spasticity}

All subjects were asked whether they currently experienced spasticity, and, if so, whether spasticity constituted a significant problem by restricting activities of daily life, by causing pain, or both. Two hundred and fourty subjects had a spastic paralysis, out of whom 99 subjects (ie $41.3 \%$ of those with spastic paralysis) reported their spasticity to be a problem.

\section{Prevalence of reported symptoms at general systems review}

Table 7 shows the prevalence of reported symptoms at a general systems review. Here, the physician went through a check-list, asking the subjects whether they had experienced specific symptoms within the last week. These symptoms are grouped according to organ systems.

\section{Bladder function}

Table 8 identifies topics related to bladder function in the study population. Problems with bladder function, taken as a whole, were graded by the subject as: 'none/minor' or 'moderate/severe'. Occurrence of uncontrolled urinary leakage was specifically sought, by asking subjects to report average frequency of leakage according to the categories: 'often' (at last every week) or 'seldom/never'. ('Uncontrolled leakage' was contrasted to 'controlled leakage', the latter examplified by non-voluntary emptying of urine through a condom catheter to a collecting device.) More than one method of urinary emptying was used by some subjects. 'Normal micturition' was defined as 'emptying by voluntary action, as before the injury'. This did not, however, exclude functional impairment, nor the use of additional methods or manoeuvers (eg external compression over lower abdomen) to achieve complete bladder emptying. 'Reflex stimulation' included tapping the lower abdomen, or related manoeuvers, to stimulate reflexogenic detrusor contraction. By contrast, 'abdominal compression' meant non-reflexogenic bladder emptying by various external compression manoeuvers.

\section{Bowel function}

Table 9 shows parameters related to bowel function. Most of the comments related to bladder function (see above) are also relevant for interpreting the bowel data. 'Bowel problems' also included constipation. Fecal leakage was grouped as 'often' (at least every week) or 'seldom/never'. Reflex versus 'passive' emptying was difficult to discriminate by questioning. 
Table 2 Causes of injury

\begin{tabular}{|c|c|c|}
\hline Cause & No. $(M / F)$ & $\%(M / F)$ \\
\hline $\begin{array}{l}\text { Transport accidents } \\
\text { Railway accidents } \\
\text { Motor vehicle traffic accidents } \\
\text { Pedal cyclist accident } \\
\text { Other road vehicle accident } \\
\text { Water transport accidents } \\
\text { Air and space transport accident }\end{array}$ & $\begin{array}{r}161(129 / 32) \\
5 \\
151 \\
1 \\
1 \\
2 \\
1\end{array}$ & $45.6(45.1 / 47.8)$ \\
\hline $\begin{array}{l}\text { Falls } \\
\text { Fall (on or) from stairs or steps } \\
\text { Fall (on or) from ladder or scaffolding } \\
\text { Fall from (out or through) building or other structure } \\
\text { Fall in a hole or other opening in the ground or floor } \\
\text { Other fall from one level to another } \\
\text { Fall on the same level through slipping, stumbling or tripping } \\
\text { Fall on the same level from a push or collision with another person } \\
\text { Unspecified cause of fall } \\
\text { Other and unspecified fall }\end{array}$ & $\begin{array}{r}131(110 / 21) \\
7 \\
12 \\
39 \\
29 \\
22 \\
7 \\
4 \\
4 \\
7\end{array}$ & $37.1(38.5 / 31.3)$ \\
\hline $\begin{array}{l}\text { Other accidents } \\
\text { Struck by thrown, projected or falling object } \\
\text { Striking against or struck by other object } \\
\text { Caught, crushed, jammed or pinched in or between objects } \\
\text { Contact with machinery } \\
\text { Discharge from firearm } \\
\text { Overexertion or strenuous or repetitive movements }\end{array}$ & $\begin{array}{r}28(23 / 5) \\
14 \\
6 \\
1 \\
1 \\
4 \\
2\end{array}$ & $7.9(8.0 / 7.5)$ \\
\hline Sequelae of external causes of morbidity or mortality & $1(1 / 0)$ & $0.3(0.3 / 0.0)$ \\
\hline $\begin{array}{l}\text { Intentional self-harm - attempted suicide or self inflicted injury } \\
\text { Intentional self-harm by jumping from a high place } \\
\text { Intentional self-harm by unspecified means }\end{array}$ & $\begin{array}{r}14(9 / 5) \\
12 \\
2\end{array}$ & $4.0(3.1 / 7.5)$ \\
\hline $\begin{array}{l}\text { Assault } \\
\text { Assault by bodily force or sexual assault by bodily force } \\
\text { Assault by rifle, shotgun and larger firearm discharge } \\
\text { Assault by sharp object }\end{array}$ & $\begin{array}{r}11(7 / 4) \\
5 \\
5 \\
1\end{array}$ & $3.1(2.4 / 6.0)$ \\
\hline $\begin{array}{l}\text { Event of undetermined intent } \\
\text { Falling, jumping or pushed from a high place, undetermined intent } \\
\text { Unspecified event, undetermined intent }\end{array}$ & $\begin{array}{l}4(4 / 0) \\
3 \\
1\end{array}$ & $1.1(1.4 / 0.0)$ \\
\hline Operations of war & $3(3 / 0)$ & $0.8(1.0 / 0.0)$ \\
\hline Total & $353(286 / 67)$ & \\
\hline
\end{tabular}

$\mathrm{M}=$ male, $\mathrm{F}=$ female

Table 3 Neurological classification

\begin{tabular}{|c|c|c|c|c|c|c|c|c|}
\hline \multirow[t]{2}{*}{ Neurological level of lesion } & \multirow[t]{2}{*}{ Total } & \multirow[t]{2}{*}{$\%$} & \multicolumn{2}{|c|}{ Complete } & \multicolumn{2}{|c|}{ Incomplete } & \multicolumn{2}{|c|}{ No information } \\
\hline & & & No. & $\%$ & No. & $\%$ & No. & $\%$ \\
\hline Cervical & 147 & 41.6 & 53 & 38.1 & 93 & 44.3 & 1 & 25.0 \\
\hline Thoracic & 127 & 36.0 & 78 & 56.1 & 48 & 22.9 & 1 & 25.0 \\
\hline Lumbar & 52 & 14.7 & 7 & 5.0 & 43 & 20.5 & 2 & 50.0 \\
\hline Sacral & 5 & 1.4 & 1 & 0.7 & 4 & 1.9 & 0 & 0.0 \\
\hline No levela & 22 & 6.2 & 0 & 0 & 22 & 10.5 & 0 & 0.0 \\
\hline Total & 353 & & 139 & & 210 & & 4 & \\
\hline
\end{tabular}

aNo residual sensorimotor deficit that makes it possible to identify the original lesion

Therefore, all methods of abdominal compression and all methods of digital manipulation of the rectum, respectively, were clustered. Bowel emptying by 'other methods' most commonly meant laxatives taken orally.

\section{Sexual function}

Tables 10 and 11 give information about key questions related to sexual function. Again, subjects were asked to grade overall perceived problems using the same 
Table 4 Medical complications after discharge from initial hospitalisation

\begin{tabular}{lrr} 
& No. & $\%$ \\
\hline Deep vein thrombosis $^{\mathrm{a}}$ & 18 & 5.1 \\
Pulmonary embolism $^{\mathrm{a}}$ & 4 & 1.1 \\
Septicaemia $^{\mathrm{a}}$ & 26 & 7.4 \\
Pneumonia $^{\mathrm{a}}$ & 31 & 8.8 \\
Decubitus ulcer $^{\mathrm{b}}$ & 137 & 38.8 \\
Urinary tract infection $^{\mathrm{c}}$ & 238 & 67.4 \\
Urolithiasis $^{\mathrm{d}}$ & 73 & 20.7 \\
Renal functional impairment $^{\mathrm{e}}$ & 22 & 6.2 \\
Fracture $^{\mathrm{a}}$ & 71 & 20.1 \\
Spinal deformity $^{\mathrm{f}}$ & 81 & 22.9 \\
Other complicationg $^{\mathrm{N}}$ & 101 & 28.6 \\
No complication & 43 & 12.2 \\
\hline
\end{tabular}

aDiagnosed by physician

bErosion of skin due to pressure, treated surgically or conservatively

cSymptomatic, verified by culture, treated with antibiotics

${ }^{\mathrm{d}}$ All locations within urinary tract, frequently but not necessarily requiring treatment

eDiagnosed by physician, all degrees of severity

fStructural or functional deformity perceived by patient and frequently verified by imaging techniques

sSee text for specification

Table 5 Progression of, and/or new neurological symptoms after discharge from initial hospitalisation

\begin{tabular}{lrr}
\hline Symptom & No. & $\%$ \\
\hline One or more symptoms & 115 & 32.6 \\
Decreased muscle power & 21 & 5.9 \\
Decreased sensation & 28 & 7.9 \\
Pain & 55 & 15.6 \\
Spasticity & 58 & 16.4 \\
Dysreflexia & 3 & 0.8 \\
Hyperhidrosis & 23 & 6.5 \\
\hline
\end{tabular}

word identifiers as those used to classify bladder and bowel function (see above). Orgasm was defined as 'a climax of sexually pleasurable sensations during intercourse or other sexual activity', therefore not necessarily related to ejaculation in the male, or even genital stimulation or sensation at all. Spontaneous erection meant 'erection occurring without either induced mechanical or psychological stimuli'. Erectile quality meant stiffness of erection, and erectile duration meant average time period of sustained erection. Subjects were asked to rate erections in terms of perceived ability to copulate.

\section{Discussion}

Accuracy of subject identification

The known drop-out rate in this study is very low. However, it is not possible to reach absolute certainty as regards the comprehensiveness of subject identification. Since current registers from all intraregional SCI outpatient services were made available, as well as registers from patient organisations and private rehabilitation facilities, it is highly unlikely that more than occasional subjects would be missed. Subjects not included in this SCI network are presumably less severely injured and less likely to have secondary medical complications. This might lead to some degree of upward bias in terms of estimated occurrence of medical problems.

The regional computerised data bank contains registration of the all-cause, total intraregional inpatient treatment since 1975. Search in this data bank by International Classification of Diseases (ICD-9) diagnoses 806 and 952 (and their equivalents in earlier editions of this classification), yielded most of the subjects primarily identified by the sources stated above, except those who had been hospitalised before 1975 and not since, and those with initial extraregional hospitalisation who later moved to the region but underwent no subsequent hospitalisation. In addition, this source yielded several subjects, where the diagnosis of traumatic SCI proved to be incorrect. This was disclosed at stage 2 of subject identification. Almost all of these subjects in reality had been hospitalised for either spinal trauma without evidence of spinal cord or cauda equina lesions, or because of spinal cord injury of non-traumatic aetiology. Whether this diagnostic inconsistency in terms of registration is unique for this region and for this disease group is unknown to us. It does point to a potential problem with register studies, if further diagnostic confirmation is not performed.

While this particular source for subject identification seemed problematic, the availability of multiple sources, as well as the subsequent confirmation of diagnosis, made anything but marginal drop-out in this study highly unlikely.

Table 6 Prevalence of significant pain and subclassification according to pain type and severity

\begin{tabular}{|c|c|c|c|c|c|c|}
\hline & \multicolumn{2}{|c|}{ Total } & \multicolumn{2}{|c|}{ Primary problem } & \multicolumn{2}{|c|}{ Not primary problem } \\
\hline & No. & $\%$ & No. & $\%$ & No. & $\%$ \\
\hline Neurogenic pain & 107 & 30.3 & 55 & 51.4 & 52 & 48.6 \\
\hline Non-neurogenic pain & 60 & 17.0 & 13 & 21.7 & 47 & 78.3 \\
\hline Both neurogenic and non-neurogenic pain & 60 & 17.0 & 34 & 56.7 & 26 & 43.3 \\
\hline No pain & 126 & 35.7 & & & & \\
\hline Total & 353 & & 102 & 28.9 & 125 & 35.5 \\
\hline
\end{tabular}


Table 7 General systems review. Numbers of reported symptoms for all subjects, and the percentage of subjects who reported one or more of these symptoms within the category headings within the last week

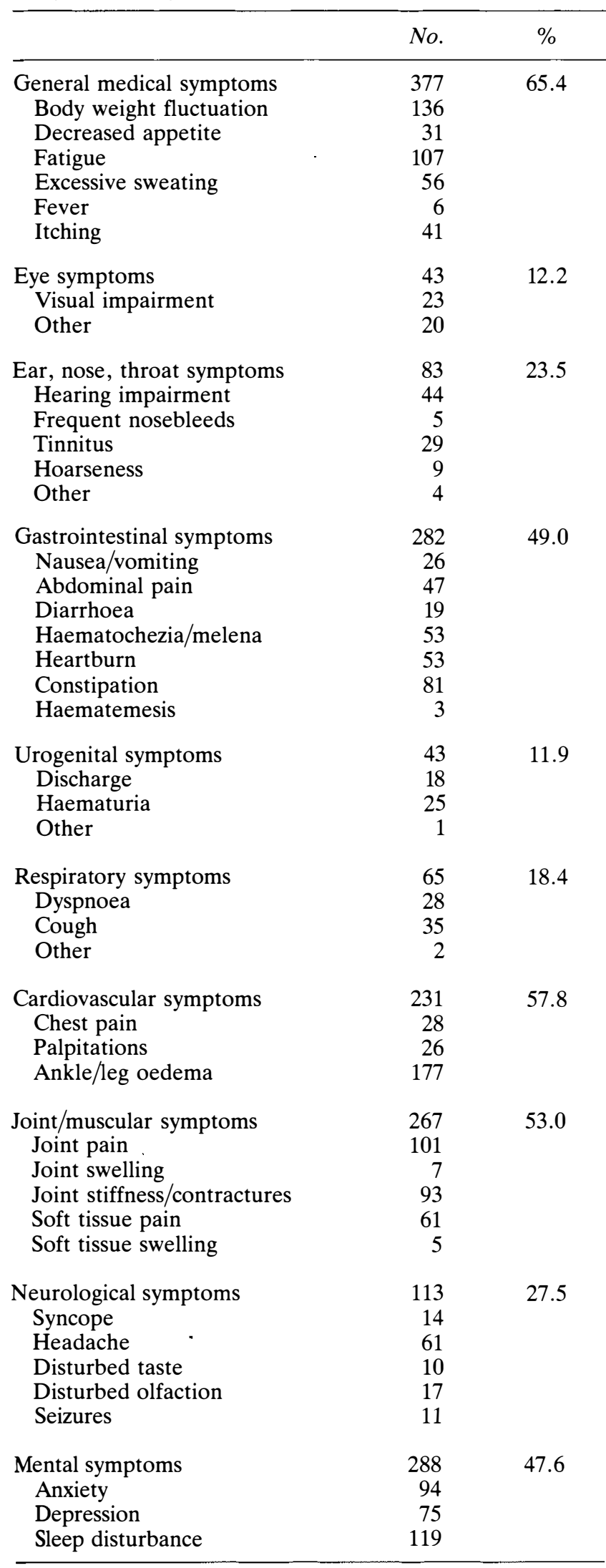

Table 8 Reported bladder function

\begin{tabular}{lrc}
\hline & No. & $\%$ \\
\hline $\begin{array}{l}\text { Subjective moderate/severe bladder } \\
\quad \text { function problem }\end{array}$ & 140 & 39.7 \\
Urine leakage often & 89 & 25.2 \\
Method of bladder emptying & & \\
$\quad$ Normal micturition & 121 & 34.3 \\
$\quad$ Intermittent catheterisation & 67 & 19.0 \\
Reflex stimulation & 109 & 30.9 \\
$\quad$ Abdominal pressure & 54 & 15.3 \\
$\quad$ Indwelling urethral catheter & 18 & 5.1 \\
$\quad$ Urinary deviation & 9 & 2.5 \\
$\quad$ Other & 35 & 9.9 \\
$\quad$ Incontinence protection & & \\
$\quad$ Condom catheter & & \\
Pad/sanitary towel & 118 & \\
\hline
\end{tabular}

aOnly male subjects ( $41.3 \%$ of all male subjects)

Table 9 Reported bowel function

\begin{tabular}{lrr} 
& No. & $\%$ \\
\hline $\begin{array}{lrr}\text { Subjective moderate/severe bowel function } \\
\quad \text { problem }\end{array}$ & 135 & 38.2 \\
Faecal leakage often & 23 & 6.5 \\
Method of bowel emptying & & \\
$\quad$ Normal defecation & 163 & 46.2 \\
$\quad$ Reflex defecation & 42 & 11.9 \\
$\quad$ Reflex/digital defecation & 92 & 26.1 \\
Suppository/enema & 106 & 30.0 \\
$\quad$ Stoma & 5 & 1.4 \\
$\quad$ Other & 82 & 23.2 \\
Incontinence protection & & \\
$\quad$ Pad/sanitary towel & 42 & 11.9 \\
Other & 6 & 1.7 \\
\hline
\end{tabular}

Accuracy of collected data

Each subject was interviewed at our clinic. The interview was performed by medical staff specialising in SCI. The interview followed a structured format, and included giving operational definitions of every potentially equivocal item. These operational definitions are stated in this paper, and should be taken into consideration when interpreting the results.

Data acquired by direct questioning alone is potentially unreliable, particularly when dealing with data many years back in time. In this study, however, previous medical records were available and used as reference in over $96 \%$ of the subjects.

\section{Interpretation of demographics}

When comparing the demographics of our study population and its similarities and dissimilarities with previous studies on SCI epidemiology, it should be remembered that our material represents a prevalence sample, whereas most previous studies are based on 
Table 10 Sexual function, males and females

\begin{tabular}{|c|c|c|c|c|c|c|}
\hline & \multicolumn{2}{|c|}{ Total } & \multicolumn{2}{|c|}{ Males } & \multicolumn{2}{|c|}{ Females } \\
\hline & No. & $\%$ & No. & $\%$ & No. & $\%$ \\
\hline Subjective moderate/severe sexual problem & 159 & 45.0 & 142 & 49.7 & 17 & 25.4 \\
\hline Orgasm altered & 97 & 27.5 & 84 & 29.4 & 13 & 19.4 \\
\hline No orgasm & 126 & 35.7 & 107 & 37.4 & 19 & 28.4 \\
\hline No sexual intercourse achieved or attempted since SCI & 98 & 27.8 & 79 & 27.6 & 19 & 28.4 \\
\hline
\end{tabular}

Table 11 SCI male subgroup. Erectile and ejaculatory function

\begin{tabular}{lrr}
\hline & \multicolumn{2}{c}{ Males } \\
\cline { 2 - 3 } & No. & $\%$ \\
\hline Erection & & \\
$\quad$ Spontaneous & 172 & 60.1 \\
Mechanical stimulation & 202 & 70.6 \\
Psychological/mental stimulation & 98 & 34.3 \\
Unable to have erection & 44 & 15.4 \\
Quality of erection & & \\
Good & 145 & 50.7 \\
$\quad$ Variable & 29 & 10.1 \\
Poor & 71 & 24.8 \\
$\quad$ Missing information & 41 & 14.3 \\
Duration of erection & & \\
$\quad$ Good & 55 & 19.2 \\
$\quad$ Variable & 62 & 21.7 \\
Poor & 95 & 33.2 \\
$\quad$ Missing information & 74 & 25.9 \\
Tried erectile aids & 35 & 12.2 \\
No ejaculation & 102 & 35.7 \\
\hline
\end{tabular}

incidence samples. Most importantly, differential survival rates in the population will influence the demographic profile, and thus preclude direct comparisons. With this in mind, it can be concluded that the expected predominance of young males injured in transport accidents and falls, with more paraplegia than tetraplegia, and more incomplete than complete lesions, is characteristic also for our study population.

\section{Interpretation of medical complications}

These results reflect the proportion of subjects within a prevalence-based SCI population that have experienced a given complication at least once since discharge from initial hospitalisation. It serves to illustrate the vulnerability of SCI patients, and thus the propensity for secondary complications involving many organ systems in the chronic stage of SCI. By definition, this study represents the experience of subjects alive at the time of the survey. A complete picture requires a prospective design, where rates and causes of mortality, as well as cumulative incidences of complications, can be ascertained.
Interpretation of medical symptoms

Neurological deterioration We find the occurrence of new and/or progressing symptoms to be unexpectedly frequent. Even though some of these symptoms are quite non-specific, our symptom descriptors reflecting neurological deterioration are similar to those experienced by confirmed cases of post-traumatic syrin $x^{12}$ and progressive post-traumatic myelomalacic myelopathy. ${ }^{13}$ Additionally, other neurological causes of deterioration, such as nerve root compression and peripheral compression neuropathies, as well as nonneurological diseases masking as neurological deterioration, should be investigated and treated if possible.

Pain and spasticity The frequent occurrence of pain among our subjects, particularly neurogenic-type pain, deserves special attention. There is a high degree of variability in the literature as regards the prevalence and severity of pain in persons with chronic SCI, although some reports have cited all-cause pain as an important problem in the management of chronic SCI. ${ }^{14-20}$ We find that both the prevalence of 'significant' pain and its classification as the 'primary problem' in our study group are remarkably high, with $64 \%$ of subjects reporting significant pain, and with $29 \%$ stating pain to be the primary problem. Over half of the subjects with neurogenic-type pain and nearly 57 percent with both neurogenic and non-neurogenic-type pain reported that pain was their primary medical problem. As intractable chronic pain may lead to drug dependency, dysphoria, suicidal ideation, ${ }^{21}$ and diminished quality of life, ${ }^{22}$ such pain merits special attention and medical follow-up.

When assessing prevalence of both pain and spasticity problems, it should be taken into account that several subjects received pharmacological and/or physical treatment at the time of the study, and that some subjects previously had also undergone surgical procedures to alleviate these problems. Thus, it is reasonable to believe that the prevalence of pain and spasticity problems is even higher, but in some instances is relieved by treatment and therefore is no longer perceived as a significant problem.

General medical symptoms Medical symptoms from most organ systems are frequent in our population. Lack of accurate data from matched controls, however, precludes the possibility at this stage to determine 
whether SCI subjects indeed experience such symptoms more or less often than the general population. Our results nevertheless point to the importance of not limiting the medical assessment of persons with SCI to, for instance, the neurological and urological systems and their complications alone.

This first study coincides with the starting point for a comprehensive, regional outpatient service for SCI patients. All subjects of this study, as well as all new cases within the region, will be followed prospectively on a yearly basis, by using protocols similar to those utilised in this study. This will enable more detailed descriptions of the long-term consequences of traumatic SCI.

\section{Acknowledgements}

This study was supported by The Spinalis Foundation and the Swedish Medical Research Council (grant 06555). The authors thank Ms Susanne Wikblad for excellent secretarial help, and Mr Petter Gustavsson for statistical assistance.

\section{References}

1 Guttmann L. Spinal Cord Injuries: Comprehensive Management and Research. 2nd edn. Blackwell Scientific Publications: Oxford, 1976.

2 Stover SL. Spinal Cord Injury: The Facts and Figures. University of Alabama at Birmingham: Birmingham, AL, 1986.

3 Stover SL, Fine PR. The epidemiology and economics of spinal cord injury. Paraplegia 1987; 25: 225-8.

4 Whiteneck GG (ed). Aging with Spinal Cord Injury. Demos Publications: New York, 1992.

5 Whiteneck GG et al. Mortality, morbidity, and psychosocial outcomes of persons spinal cord injured more than 20 years ago. Paraplegia 1992; 30: 617-30.

6 Parsons KC, Lammertse DP. Rehabilitation in spinal cord disorders. 1. Epidemiology, prevention, and system of care of spinal cord disorders. Arch Phys Med Rehabil 1991; 72: S293-4.

7 Burney RE, Maio RF, Maynard F, Karunas R. Incidence, characteristics, and outcome of spinal cord injury at trauma centers in North America. Arch Surg 1993; 128: 596-9.

8 Dialogdata Information Systems Ltd, Stockholm, Sweden. INREGIA Institute for Regional Analysis, Sweden, 1991.

9 Levi R, Hultling C, Westgren N. A computer assisted follow-up system for spinal cord injury patients. Paraplegia 1994; 32: 736-742.

10 Ditunno JF, Young W, Donovan WH, Creasey G. The international standards booklet of neurological and functional classification of spinal cord injury. Paraplegia 1994; 32: 70-80.

11 Klassifikation Av Sjukdomar, Systematisk Förteckning (International Classification of Diseases, Ninth Revision ICD-9). Swedish version, 1987.

12 Edgar R, Quail P. Progressive post-traumatic cystic and noncystic myelopathy. Br J Neurosurg 1994; 8: 7-22.

13 Falcone $\mathrm{S}$ et al. Progressive posttraumatic myelomalacic myelopathy: imaging and clinical features. Am J Neuroradiol 1994; 15: $747-54$

14 Fenollosa $\mathrm{P}$ et al. Chronic pain in the spinal cord injured: Statistical approach and pharmacological treatment. Paraplegia 1993; 31: 722-9.

15 Svendsen L, Drewes AM, Biering-Sorensen F, Lonnberg F. Chronic pain after spinal cord injury-a descriptive study. Ugeskr Laeger 1993; 155: 3118-22.

16 Mariano AJ. Chronic pain and spinal cord injury. Clin J Pain 1992; 8: 87-92.

17 Balazy TE. Clinical management of chronic pain in spinal cord injury. Clin J Pain 1992; 8: 102-10.

18 Kennedy RH. A new viewpoint toward spinal cord injuries. Ann Surg 1946; 124: 1057-1065.

i9 Botetrel EH, Callaghan JC, Jousse AT. Pain in paraplegia: Clinical management and surgical treatment. Proc R Soc Med 1953; 47: 281-288.

20 Nepomuceno C, Fine PR, Richards JS. Pain in patients with spinal cord injury. Arch Phys Med Rehabil 1979; 60: 605-609.

21 Rapaport MH. Chronic pain and posttraumatic stress disorder. Am J Psychiatry 1987; 144: 120.

22 Siösteen A. Adjustment to spinal cord injury: A clinical and experimenial study on quality of life, sexuality and fertility. (Doctoral thesis) Departments of Neurosurgery, Neurology and Medicine I/Health Care Research Unit, University of Göteborg, Göteborg, Siveden, 1989. 